\title{
Delay to formalin fixation 'cold ischemia time': effect on ERBB2 detection by in-situ hybridization and immunohistochemistry
}

\author{
Bryce P Portier ${ }^{1}$, Zhen Wang ${ }^{1}$, Erinn Downs-Kelly ${ }^{1}$, Jordi J Rowe ${ }^{1}$, Deepa Patil ${ }^{1}$,
} Chis Lanigan ${ }^{1}$, G Thomas Budd ${ }^{2}$, David G Hicks ${ }^{3}$, David L Rimm ${ }^{4}$ and Raymond R Tubbs ${ }^{1}$

${ }^{1}$ Pathology and Laboratory Medicine Institute, Cleveland Clinic, Cleveland, OH, USA; ${ }^{2}$ Department of Solid Tumor Oncology, Taussig Cancer Institute, Cleveland Clinic, Cleveland, OH, USA; ${ }^{3}$ Department of Pathology and Laboratory Medicine, University of Rochester, Rochester, NY, USA and ${ }^{4}$ Department of Pathology,

Yale University, New Haven, CT, USA

\begin{abstract}
The American Society of Clinical Oncology/College of American Pathologists ERBB2 testing guidelines address several pre-analytical variables known to affect ERBB2 testing accuracy. According to 2010 updated guidelines, the pre-analytical variable of time to tissue fixation (cold ischemia time) should be kept to $<1 \mathrm{~h}$, however, little has been published about cold ischemia time and its significance in ERBB2 testing. To that end, this study evaluated ERBB2 status using two different FDA-approved in-situ hybridization methods and an FDA-approved immunohistochemistry $(\mathrm{IHC})$ assay in the largest cohort to date $(n=84)$ of invasive breast carcinomas with tracked cold ischemia time. Cold ischemia time was stratified into four groups $(<1 \mathrm{~h}(n=45), 1-2 \mathrm{~h}(n=27)$, 2-3h $(n=6)$, and $>3 h(n=6))$ and ERBB2 status was evaluated in each group by IHC (4B5) and by in-situ hybridization methodologies (PathVysion ${ }^{\circledR}$ fluorescence in situ hybridization and the INFORM HER2 ${ }^{\circledR}$ dual in situ DNA probe assay). Both in-situ hybridization methods were evaluated using three ERBB2 scoring criteria (dual-probe guidelines, single-probe guidelines, and the FDA package insert scoring instructions). Fluorescence in-situ hybridization (FISH) and INFORM HER2 ${ }^{\circledR}$ demonstrated $100 \%$ concordance in the detection of ERBB2 amplification by all three scoring guidelines at all cold ischemia time points. Agreement between in-situ hybridization methodologies and IHC was superior using single-probe guidelines compared with dual probe or FDA scoring instructions. In addition, Inform HER2 in-situ hybridization signals were significantly more intense than FISH at all cold ischemia time points, however, no significant loss of either chromosome 17 or ERBB2 signal was detected by FISH or Inform HER2 ${ }^{\circledR}$ in-situ hybridization in cold ischemia times up to $3 \mathrm{~h}$. On the basis of our findings, cold ischemia time up to $3 \mathrm{~h}$ has no deleterious effect on the detection of ERBB2 via in-situ hybridization or IHC.
\end{abstract}

Modern Pathology (2013) 26, 1-9; doi:10.1038/modpathol.2012.123; published online 17 August 2012

Keywords: breast carcinoma; cold ischemia time; ERBB2; fluorescence in-situ hybridization; HER2; inform HER2 dual in-situ hybridization; trastuzumab

The ability to perform accurate initial ERBB2 testing is absolutely critical as this laboratory determination directly impacts clinical decision making and patient management. ERBB2 is more commonly referred to as HER2, however, ERBB2 is the official name designated by the HUGO Gene Nomenclature Committee and will be used throughout the

Correspondence: Professor RR Tubbs, DO, Department of Pathology and Laboratory Medicine, Cleveland Clinic, 9500 Euclid Avenue LL2, Cleveland, OH, USA.

E-mail: tubbsr@ccf.org

Received 26 January 2012; revised 23 May 2012; accepted 23 May 2012; published online 17 August 2012 manuscript. The downstream clinical result of accurate determination of ERBB2 status is administration of trastuzumab therapy to those patients that have amplified or overexpressed ERBB2. ${ }^{1-3}$ Therefore, accurate stratification of patients into ERBB2-positive or -negative results in increasing the likelihood of a positive response to drug therapy, ${ }^{1,4,5}$ limits unnecessary exposure to the drugs side effects (in particular the risk of cardiac sequelae), ${ }^{6-8}$ and prevents substantial unnecessary expense. ${ }^{9-11}$ In multiple studies, trastuzumab therapy has shown to improve progression-free survival and overall survival, when added to standard chemotherapy regimens in both the metastatic and adjuvant 
settings. ${ }^{1,3-5}$ The American Society of Clinical Oncology/College of American Pathologists (ASCO/ CAP) jointly addressed the challenging task of establishing ERBB2 testing guidelines that have set a standard for improving the accuracy of testing via both immunohistochemistry (IHC) and in-situ hybridization (ISH) methodologies. ${ }^{12,13}$ These guidelines consider pre-analytic, analytic, and post-analytic variables that directly affect testing accuracy.

Several studies have addressed analytical and post-analytical variables in ERBB2 testing. ${ }^{14-21}$ However, a limited number of studies, with small patient sample sizes, have been performed to specifically address the effect of the pre-analytical variable of delayed formalin fixation (or cold ischemia time) and its effect on ERBB2 detection. ${ }^{18,22}$ In regard to pre-analytical variables, the ASCO/CAP guidelines recommend fixation with $10 \%$ neutral-buffered formalin and a length of fixation $>6$ but $<48 \mathrm{~h}^{12}$ In addressing the preanalytical variable of cold ischemia time, the initial 2007 ASCO/CAP guidelines recommended keeping the cold ischemia time as brief as possible. ${ }^{12}$ However, current ASCO/CAP guidelines (April 2010 update) now recommend cold ischemia time in ERBB2 testing be maintained at $<1 \mathrm{~h} .{ }^{13}$ Changes to the new guidelines were heavily dependent on the findings of a single study by Khoury et $a{ }^{18}{ }^{18}$ which investigated IHC and fluorescence in-situ hybridization (FISH) results in one ERBB2-positive case following increasing cold ischemia time. Khoury et al reported that ERBB2 IHC retained staining at all cold ischemia time points; however, the FISH assay was compromised after $1 \mathrm{~h}$ of cold ischemia time, with progressive loss of evaluable cases with longer cold ischemia time. Recently, a publication by Moatomed et al demonstrated findings that are contrary to the Khoury et al with respect to ERBB2 FISH results and cold ischemia time. In the Moatamed et $a l^{22}$ study, cold ischemia time was varied from $<1 \mathrm{~h}$ up to 4 days with tissue stored at $4{ }^{\circ} \mathrm{C}$; they found no loss of FISH signal. With only two contradictory publications evaluating cold ischemia time effect on ERBB2 testing, and the supplemental guideline based upon limited literature, there is currently a lack of evidencebased results.

An additional variable that complicates ERBB2 testing by ISH is that there are three different published scoring criteria (ASCO/CAP dual-probe ratio, ASCO/CAP single ERBB2 probe guideline, and the FDA package insert scoring instructions). ${ }^{12}$ In this study, we specifically address the role of cold ischemia time on ERBB2 status determination and examine the effect of utilizing different ERBB2 scoring guidelines, in the context of three different detection methods including IHC, interphase FISH, and the newly FDA-approved bright field microscopy method of INFORM HER2 ${ }^{\circledR}$ dual in situ hybridization assay (DDISH).

\section{Material and methods}

\section{Patient Population}

A total of 92 patients with a primary diagnosis of invasive breast carcinoma were identified from the Department of Pathology and Laboratory Medicine at the University of Rochester (collected between August 2008 and August 2009). For this study, resection specimens were used wherein both the time of resection from the patient (time the specimen was handed off the operative field) and the time the sample was placed in 10\% neutral-buffered formalin were accurately recorded, representing the cold ischemia time. The cold ischemia time ranged from $27 \mathrm{~min}$ to $6: 25$ (h: $\mathrm{min})$. Cold ischemia time of samples were representative of actual practice times to fixation; specimen handling and logistics were not altered for this study. ${ }^{23}$ Of the 92 cases, eight cores were excluded from the study (seven contained no tumor and one core contained only ductal carcinoma in-situ). The evaluable cold ischemia time population $(n=84)$ segregated as follows: $<1 \mathrm{~h}(n=45), 1-2 \mathrm{~h}(n=27), 2-3 \mathrm{~h}(n=6)$, and $>3 \mathrm{~h} \quad(n=6)$. The $>3 \mathrm{~h}$ cold ischemia time population consisted of six cases with a cold ischemia time ranging from 3:27-6:25 (h: $\mathrm{min}$ ) $(3: 27,3: 30,4: 32,5: 25,5: 28$, and 6:25).

\section{Tissue Microarray}

Tissue microarrays (TMAs) including duplicate 0.6$\mathrm{mm}$ cores of invasive breast carcinoma with accurately tracked cold ischemia time were constructed at the Yale University Department of Pathology utilizing formalin-fixed, paraffin-embedded tissue of 93 breast cancer patients, who had surgery at the University of Rochester, School of Medicine, (Rochester, NY). A TMA was constructed, consisting of these 93 breast cancer specimens, and a small series of cell lines and control tissues, all represented in twofold redundancy. Following processing, 84 cases had at least one core with invasive tumor that could be scored. Twelve cases lost their mated pair core between TMA \#1 and \#2, resulting in 72 cores with a mated pair and 12 cores lacking a mated pair.

\section{ERBB2 Testing and Scoring}

ERBB2 status determination was performed on the TMAs utilizing IHC with the FDA-approved 4B5 rabbit monoclonal antibody (Ventana Medical Systems, Tucson, AZ), an FDA-approved interphase FISH assay (PathVysion ${ }^{\circledR}$, Abbott Molecular, Des Plaines, IL), and the recently FDA-approved bright field microscopy method of INFORM HER2 ${ }^{\circledR}$ dual in situ hybridization, which is a dual color, dual hapten DNA in situ hybridization assay (DDISH) (Ventana Medical Systems, Tucson, AZ). ERBB2 scoring methods were applied to FISH and DDISH 
samples in accordance to published ASCO/CAP guidelines and the FDA package insert. ${ }^{12}$ Briefly, ASCO/CAP dual-probe scoring was applied as follows: non-amplified ( $E R B B 2 / \mathrm{CEP} 17<1.8)$, equivocal (ERBB2/CEP17 1.8-2.2), or amplified (ERBB2/ CEP17 > 2.2). Scoring following ASCO/CAP singleprobe guidelines were as follows: amplified $>6$ $E R B B 2$ signals, equivocal between 4 and 6 ERBB2 signals, and non-amplified $<4$ ERBB2 signals. Scoring following the FDA in-situ hybridization package insert were as follows: amplified $E R B B 2 /$ CEP17 ratio $\geqslant 2.0$ and non-amplified ERBB2/CEP17 ratio $<2.0$. These scoring systems were applied to FISH and DDISH following signal enumeration by three pathologists independently (EDK, JJR, and BPP). ERBB2 IHC was scored according to the ASCO/CAP guidelines as $0,1+, 2+$, or $3+.{ }^{12}$ Specifically, two pathologists reviewed each core separately and after completion of scoring, results were compared. In discordant cases, a third pathologist that was blinded to the results scored the discrepant cores. In those cases, the final result was recorded with agreement between two pathologists. For the in situ hybridization studies, of the 72 cores with a mated pair, one core was scored counting 20 cells per core with the average ERBB2 and CEP17 results recorded. In the mated pair, all 72 cases were evaluated by scoring 10 cells per core. Mated pair scores failed to result in a change in amplified, equivocal, or non-amplified status. Therefore, the scores of the 20 cell count were utilized for result reporting.

\section{In-situ Hybridization (ISH) Signal Intensity Scoring}

The signal intensity in the FISH and DDISH assays was scored utilizing a four point system: $0=$ no signals visible, $1=$ weak signals barely visible, $2=$ signals visible but not intense, and $3=$ intense signals. This four point scoring system was applied to $E R B B 2$ and CEP17 signals in both tumor and stromal cells at each cold ischemia time. Signal intensity was evaluated independently by three pathologists (EDK, JJR, and BPP). Of note, the CEP17 signal intensity score was utilized to critically assess for the integrity of internal control signals within stromal cell nuclei and served as an index of successful hybridization and target preservation with increasing cold ischemia time.

\section{Statistics}

A two-sample $t$-test was performed to determine whether there was a significant difference between DDISH and FISH signal intensity scores for ERBB2 and CEP17 signals in both stromal and tumor cells. A $P$-value of $\leq 0.05$ was considered significant. A Mann-Whitney rank sum test was performed to determine whether there was a significant difference between in-situ hybridization signal intensity and increasing cold ischemia time. Statistical analysis was performed using GraphPad Prism V5 (GraphPad Software, La Jolla, CA) and SigmaPlot V12 (Systat Software, San Jose, CA).

\section{Results}

The cold ischemia time population segregated as follows: $<1 \mathrm{~h}(n=45), 1-2 \mathrm{~h}(n=27), 2-3 \mathrm{~h}(n=6)$, and $>3 \mathrm{~h}(n=6)$. Representative H\&E, IHC, FISH, and DDISH images for each cold ischemia time are shown in Figure 1. The results described below focus on comparing multiple variables including three ERBB2 detection methodologies, four ERBB2 scoring methods, and four cold ischemia time populations. Although emphasis is given to cases with discrepancies resulting in a change in ERBB2 status that would directly translate to changes in treatment recommendations (Table 1), cases with minor discrepancies (ie, equivocal versus nonamplified) will also be addressed (Table 2). Actual values for ISH and IHC scoring are supplied as supplemental data (Supplementary Table 1 and Supplementary Table 2).

\section{Cold Ischemia Time $<1 \mathrm{~h}$ Population}

Utilizing both ASCO/CAP dual probe and the FDA in-situ hybridization package insert scoring instructions, ERBB2 amplification was detected in four cases by FISH and DDISH (100\% concordance) (Table 1). In these four ISH-amplified cases, only one case demonstrated ERBB2 $3+$ overexpression by IHC (25\% concordance with ISH methods) (Table 1). However, FISH single-probe ASCO/CAP guidelines showed $100 \%$ agreement with IHC (Table 1). A total of eleven cases were identified in the $<1 \mathrm{~h}$ cold ischemia time group with a minor discrepant ERBB2 IHC and ISH result (alteration in equivocal and non-amplified status), however, none of these discrepancies would result in a change in ERBB2 status (Table 2).

By ISH, ERBB2 and CEP17 signals were detected in stromal cells on the same slide adjacent to scored tumor cells. The average signal intensity score for $E R B B 2$ in stromal cells was 2.8 with a s.d. $=0.4$ by DDISH and 1.8 s.d. $=0.5$ by FISH, $(P=<0.05)$ (Figure 2a). The average signal intensity score for CEP17 in stromal cells was 2.8 s.d. $=0.4$ by DDISH and 2.2 s.d. $=0.5$ by FISH, $(P=<0.05)$ (Figure $2 b)$.

\section{Cold Ischemia Time 1-2 h Population}

Within the 1-2 h cold ischemia time cohort, all three ISH ERBB2 scoring methods and IHC demonstrated $100 \%$ concordance in identifying three cases as amplified and $3+$, respectively (Table 1). A total of three cases were identified in the $1-2 \mathrm{~h}$ cold ischemia time group with a minor discrepant ERBB2 


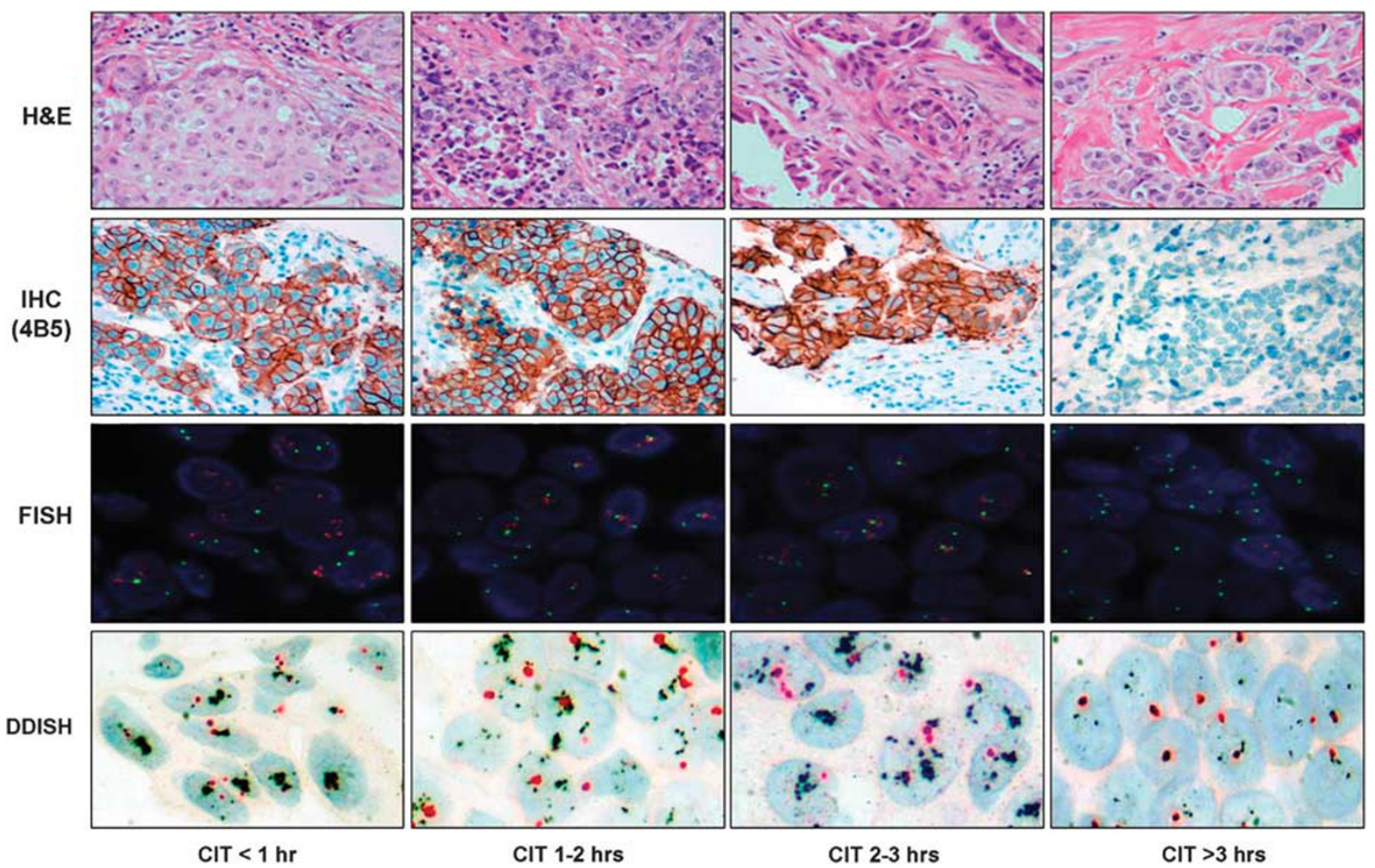

Figure 1 Representative H\&E, immunohistochemistry, fluorescence in-situ hybridization and Inform HER2 ${ }^{\circledR}$ dual in-situ hybridization images at four cold ischemia time points.

Table 1 Cases demonstrating ERBB2-amplified/overexpressed status as determined by one or more scoring methods including: ASCO/ CAP Guidelines for ISH (dual and single probe), FDA package insert scoring instructions, and ASCO/CAP Guidelines for IHC scoring

\begin{tabular}{|c|c|c|c|c|c|c|c|}
\hline \multirow{2}{*}{ CIT } & \multicolumn{3}{|c|}{ FISH } & \multirow[b]{2}{*}{$\begin{array}{c}A S C O / C A P \\
I H C\end{array}$} & \multicolumn{3}{|c|}{ DDISH } \\
\hline & $\begin{array}{c}\text { ASCO/CAP } \\
\text { (dual probe) }\end{array}$ & $\begin{array}{c}\text { FDA package } \\
\text { insert }\end{array}$ & $\begin{array}{c}\text { ASCO/CAP } \\
\text { (single probe) }\end{array}$ & & $\begin{array}{l}\text { ASCO/CAP } \\
\text { (dual probe) }\end{array}$ & $\begin{array}{c}\text { FDA package } \\
\text { insert }\end{array}$ & $\begin{array}{c}\text { ASCO/CAP } \\
\text { (single probe) }\end{array}$ \\
\hline \multicolumn{8}{|l|}{$<1 H$} \\
\hline Case 1 & A & A & A & A & A & A & A \\
\hline Case 2 & A & A & $\mathrm{E}$ & $\mathrm{E}$ & A & A & $\mathrm{N}$ \\
\hline Case 3 & A & A & $\mathrm{N}$ & $\mathrm{N}$ & A & A & $\mathrm{N}$ \\
\hline Case 4 & A & A & $\mathrm{N}$ & $\mathrm{N}$ & A & A & $\mathrm{N}$ \\
\hline \multicolumn{8}{|l|}{$1-2 H$} \\
\hline Case 1 & A & A & A & A & A & A & A \\
\hline Case 2 & A & A & A & A & A & A & A \\
\hline Case 3 & A & A & $\mathrm{A}$ & A & $\mathrm{A}$ & $\mathrm{A}$ & A \\
\hline \multicolumn{8}{|l|}{$2-3 H$} \\
\hline Case 1 & A & A & A & $\mathrm{E}$ & A & A & A \\
\hline Case 2 & A & A & A & $\mathrm{A}$ & A & A & A \\
\hline
\end{tabular}

Abbreviations: $\mathrm{A}=$ amplified/overexpressed, $\mathrm{E}=$ equivocal, $\mathrm{N}=$ non-amplified/not overexpressed.

IHC and ISH result, however, none of these discrepancies would result in a change in ERBB2 status (Table 2).

By ISH, ERBB2 and CEP17 signals were detected in stromal cells on the same slide adjacent to scored tumor cells. The average signal intensity score for
$E R B B 2$ in stromal cells was 2.8 s.d. $=0.5$ by DDISH and 1.9 s.d. $=0.5$ by FISH, $(P=<0.05)$ (Figure 2a). The average signal intensity score for CEP17 in stromal cells was 2.7 s.d. $=0.5$ by DDISH and 2.2 s.d. $=0.4$ by FISH hybridization, $(P=<0.05)$ (Figure 2b). 
Table 2 Cases demonstrating a minor discrepancy in ERBB2 scoring (alteration in equivocal and non-amplified status) as determined by one or more scoring methods including: ASCO/CAP Guidelines for ISH (dual and single probe), FDA package insert scoring instructions, and ASCO/CAP Guidelines for IHC scoring

\begin{tabular}{|c|c|c|c|c|c|c|c|}
\hline \multirow{2}{*}{ CIT } & \multicolumn{3}{|c|}{ FISH } & \multirow[b]{2}{*}{$\begin{array}{c}A S C O / C A P \\
I H C\end{array}$} & \multicolumn{3}{|c|}{ DDISH } \\
\hline & $\begin{array}{l}\text { ASCO/CAP } \\
\text { (dual probe) }\end{array}$ & $\begin{array}{c}\text { FDA package } \\
\text { insert }\end{array}$ & $\begin{array}{c}\text { ASCO/CAP } \\
\text { (single probe) }\end{array}$ & & $\begin{array}{l}\text { ASCO/CAP } \\
\text { (dual probe) }\end{array}$ & $\begin{array}{c}\text { FDA package } \\
\text { insert }\end{array}$ & $\begin{array}{c}\text { ASCO/CAP } \\
\text { (single probe) }\end{array}$ \\
\hline \multicolumn{8}{|l|}{$<1 H$} \\
\hline Case 1 & $\mathrm{E}$ & $\mathrm{N}$ & $\mathrm{N}$ & $\mathrm{N}$ & $\mathrm{E}$ & $\mathrm{N}$ & $\mathrm{N}$ \\
\hline Case 2 & $\mathrm{~N}$ & $\mathrm{~N}$ & $\mathrm{~N}$ & $\mathrm{E}$ & $\mathrm{N}$ & $\mathrm{N}$ & $\mathrm{N}$ \\
\hline Case 3 & $\mathrm{~N}$ & $\mathrm{~N}$ & $\mathrm{~N}$ & $\mathrm{E}$ & $\mathrm{N}$ & $\mathrm{N}$ & $\mathrm{N}$ \\
\hline Case 4 & $\mathrm{~N}$ & $\mathrm{~N}$ & $\mathrm{~N}$ & $\mathrm{E}$ & $\mathrm{N}$ & $\mathrm{N}$ & $\mathrm{N}$ \\
\hline Case 5 & $\mathrm{~N}$ & $\mathrm{~N}$ & $\mathrm{~N}$ & $\mathrm{E}$ & $\mathrm{N}$ & $\mathrm{N}$ & $\mathrm{N}$ \\
\hline Case 6 & $\mathrm{~N}$ & $\mathrm{~N}$ & $\mathrm{~N}$ & $\mathrm{E}$ & $\mathrm{N}$ & $\mathrm{N}$ & $\mathrm{N}$ \\
\hline Case 7 & $\mathrm{~N}$ & $\mathrm{~N}$ & $\mathrm{~N}$ & $\mathrm{E}$ & $\mathrm{N}$ & $\mathrm{N}$ & $\mathrm{N}$ \\
\hline Case 8 & $\mathrm{~N}$ & $\mathrm{~N}$ & $\mathrm{~N}$ & $\mathrm{E}$ & $\mathrm{N}$ & $\mathrm{N}$ & $\mathrm{N}$ \\
\hline Case 9 & $\mathrm{~N}$ & $\mathrm{~N}$ & $\mathrm{~N}$ & $\mathrm{E}$ & $\mathrm{N}$ & $\mathrm{N}$ & $\mathrm{N}$ \\
\hline Case 10 & $\mathrm{~N}$ & $\mathrm{~N}$ & $\mathrm{~N}$ & $\mathrm{~N}$ & $\mathrm{~N}$ & $\mathrm{~N}$ & $\mathrm{E}$ \\
\hline Case 11 & $\mathrm{~N}$ & $\mathrm{~N}$ & $\mathrm{~N}$ & $\mathrm{~N}$ & $\mathrm{~N}$ & $\mathrm{~N}$ & $\mathrm{E}$ \\
\hline \multicolumn{8}{|l|}{$1-2 H$} \\
\hline Case 1 & $\mathrm{~N}$ & $\mathrm{~N}$ & $\mathrm{E}$ & $\mathrm{E}$ & $\mathrm{N}$ & $\mathrm{N}$ & $\mathrm{E}$ \\
\hline Case 2 & $\mathrm{~N}$ & $\mathrm{~N}$ & $\mathrm{~N}$ & $\mathrm{E}$ & $\mathrm{N}$ & $\mathrm{N}$ & $\mathrm{N}$ \\
\hline Case 3 & $\mathrm{~N}$ & $\mathrm{~N}$ & $\mathrm{~N}$ & $\mathrm{E}$ & $\mathrm{N}$ & $\mathrm{N}$ & $\mathrm{N}$ \\
\hline \multicolumn{8}{|l|}{$2-3 h$} \\
\hline Case 1 & $\mathrm{~N}$ & $\mathrm{~N}$ & $\mathrm{~N}$ & $\mathrm{E}$ & $\mathrm{N}$ & $\mathrm{N}$ & $\mathrm{N}$ \\
\hline \multicolumn{8}{|l|}{$>3 H$} \\
\hline Case 1 & $\mathrm{~N}$ & $\mathrm{~N}$ & $\mathrm{~N}$ & $\mathrm{E}$ & $\mathrm{N}$ & $\mathrm{N}$ & $\mathrm{N}$ \\
\hline Case 2 & $\mathrm{~N}$ & $\mathrm{~N}$ & $\mathrm{~N}$ & $\mathrm{E}$ & $\mathrm{N}$ & $\mathrm{N}$ & $\mathrm{N}$ \\
\hline Case 3 & $\mathrm{~N}$ & $\mathrm{~N}$ & $\mathrm{~N}$ & $\mathrm{E}$ & $\mathrm{N}$ & $\mathrm{N}$ & $\mathrm{N}$ \\
\hline
\end{tabular}

Abbreviations: $\mathrm{A}=$ amplified/overexpressed, $\mathrm{E}=$ equivocal, $\mathrm{N}=$ non-amplified/not overexpressed.

\section{Cold Ischemia Time 2-3 h Population}

Within the 2-3 h cold ischemia time cohort, all three ISH ERBB2 scoring guidelines were consistent in identifying two ERBB2-amplified cases (Table 1). In these two ISH-amplified cases, IHC identified one case as positive $(3+)$ and one case as equivocal $(2+)$ (Table 1). Only one case was identified in the $2-3 \mathrm{~h}$ cold ischemia time group with a minor discrepant ERBB2 IHC and ISH result, however, this discrepancies would not result in a change in ERBB2 status (Table 2).

By ISH, ERBB2 and CEP17 signals were detected in stromal cells on the same slide adjacent to scored tumor cells. The average signal intensity score for $E R B B 2$ in stromal cells was 2.5 s.d. $=0.8$ by DDISH and 1.5 s.d. $=0.5$ by FISH, $(P=<0.05)$ (Figure 2a). The average signal intensity score for CEP17 in stromal cells was 2.7 s.d. $=0.5$ by DDISH and 1.8 s.d. $=0.8$ by FISH, $(P=<0.05)$ (Figure $2 b)$.

\section{Cold Ischemia Time $>3 \mathrm{~h}$ Population}

Within the $>3 \mathrm{~h}$ cold ischemia time cohort, all three ISH scoring guidelines showed 100\% concordance and no amplified cases were identified by ISH or IHC. A total of three cases were identified in the $>3 \mathrm{~h}$ cold ischemia time group with a minor discrepant ERBB2 IHC and ISH result, however, none of these discrepancies would result in a change in ERBB2 status (Table 2).

By ISH, ERBB2 and CEP17 signals were detected in stromal cells on the same slide adjacent to scored tumor cells. The average signal intensity score for $E R B B 2$ in stromal cells was 2.5 s.d. $=0.8$ by DDISH and 1.2 s.d. $=0.4$ by FISH, $(P=<0.05)$ (Figure 2a). The average signal intensity score for CEP17 in stromal cells was 2.5 s.d. $=0.5$ by DDISH and 1.5 s.d. $=0.5$ by FISH, $(P=<0.05)$ (Figure $2 b)$.

\section{In-situ Hybridization Signal Intensity and Preservation in Tumor Cells}

By FISH, the signal intensity for ERBB2 in tumor cells per cold ischemia time group was $<1 \mathrm{~h}: 1.8$ s.d. $=0.5,1-2$ h: 1.9 s.d. $=0.5,2-3$ h: 1.7 s.d. $=0.8$, and $>3$ h: 1.5 s.d. $=0.5$ (Figure 2c). By FISH, the signal intensity for CEP17 in tumor cells per cold ischemia time group was $<1 \mathrm{~h}: 2.3 \mathrm{s.d} .=0.5,1-2 \mathrm{~h}$ : 2.3 s.d. $=0.5,2-3 \mathrm{~h}: 1.8$ s.d. $=0.8$, and $>3 \mathrm{~h}: 1.7$ s.d. $=0.8$ (Figure 2d). In comparison to FISH, the DDISH signal intensity for ERBB2 in tumor cells per cold ischemia time group was $<1 \mathrm{~h}: 2.8 \mathrm{~s} . \mathrm{d} .=0.4$, 1-2 h: 2.7 s.d. $=0.4,2-3$ h: 2.5 s.d. $=0.5$, and $>3$ h: 2.5 s.d. $=0.5$ (Figure 2c). By DDISH, the signal 


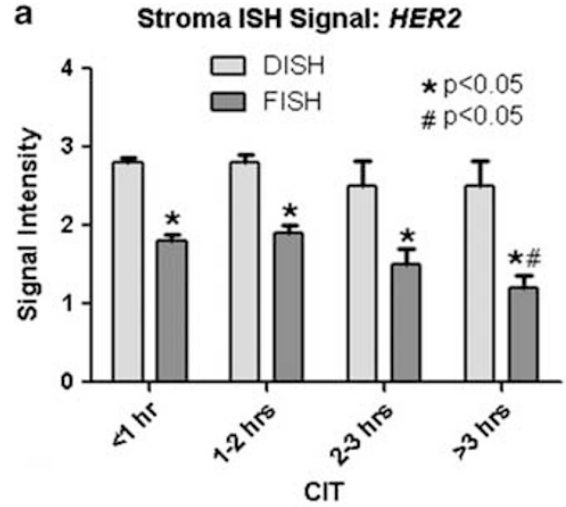

C

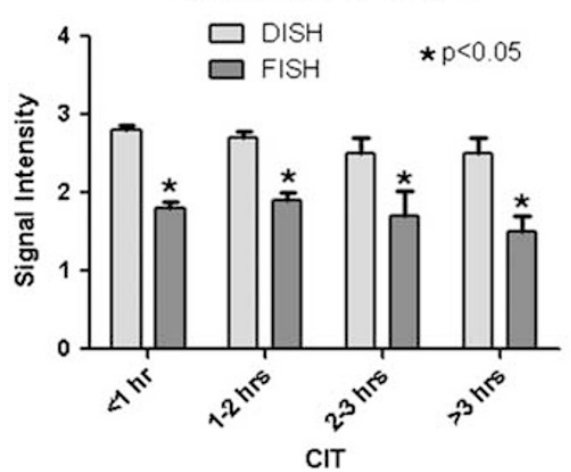

$0=$ No signals visible $1=$ Weak signals barely visible b

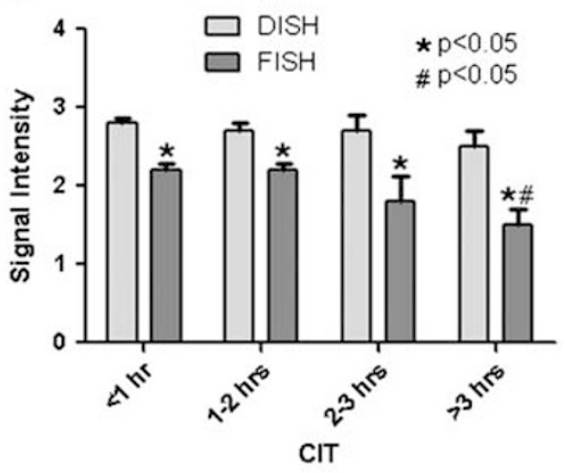

d Tumor ISH Signal: Chromosome 17

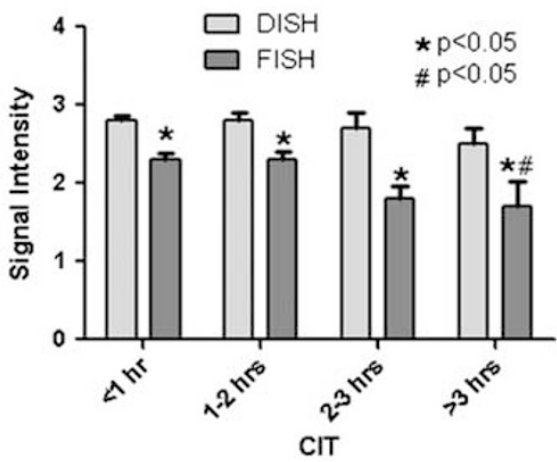

$2=$ Signals visible but not intense

$3=$ Intense signals

Figure 2 Comparison of signal intensity between Inform $H E R 2^{\circledR}$ dual in-situ hybridization and fluorescence in-situ hybridization in both stroma and tumor cells for ERBB2 and chromosome 17. *Significant difference between Inform HER2 ${ }^{\circledR}$ dual in-situ hybridization and fluorescence in-situ hybridization at specified cold ischemia time point. \#Significant difference in intra-assay signal intensity, reference signal is $<1 \mathrm{~h}$ cold ischemia time.

intensity for CEP17 in tumor cells per cold ischemia time group was $<1 \mathrm{hr}: 2.8$ s.d. $=0.4,1-2 \mathrm{~h}: 2.8$ s.d. $=0.5,2-3 \mathrm{~h}: 2.7$ s.d. $=0.5$, and $>3 \mathrm{~h}: 2.5$ s.d. $=0.5$ (Figure 2d). In comparing FISH to DDISH, there was a significant difference in the signal intensity at all cold ischemia time points $(P=<0.05)$ (Figures 2a-d). In comparing each ISH assay individually, there was no significant degradation of signal intensity with cold ischemia time up to $3 \mathrm{~h}$. However, FISH showed a significant degradation in signal intensity at $>3 \mathrm{~h}$ cold ischemia time in detecting ERBB2 and CEP17 in the stroma, as well as CEP17 in the tumor $(P=<0.05)$ (Figures 2a, b, and d).

\section{Discussion}

In this study, we compared the effect of cold ischemia time on standard ERBB2 testing methodologies including FISH and IHC along with the recently FDA-approved method of Inform HER2 ${ }^{\text {B }}$ dual in-situ hybridization. In addition, we applied three different scoring systems (ASCO/CAP dual- probe in-situ hybridization guidelines, ASCO/CAP single-probe in-situ hybridization guidelines, and the FDA in-situ hybridization package insert scoring instructions) to cases with prolonged cold ischemia time. The three methodologies and scoring guidelines were applied to a patient population $(n=84)$ with accurately tracked cold ischemia time in real-time practice at a large university teaching hospital. ${ }^{23}$ Comparison of in-situ hybridization methods (FISH and DDISH) showed no difference in detection of ERBB2-amplified cases at any cold ischemia time by ASCO/CAP dual-probe or FDA in-situ hybridization ERBB2 scoring methods. In contrast to the high concordance achieved by ISH methodologies, IHC did not identify several in-situ hybridization ERBB2-amplified cases at multiple cold ischemia times. Specifically, IHC was negative in four of nine ERBB2-amplified cases (three within the cold ischemia time $<1 \mathrm{~h}$ and one within the cold ischemia time $2-3 \mathrm{~h}$ ) as detected by ASCO/CAP dual-probe guidelines or FDA in-situ hybridization package insert scoring instructions. In these four cases, it is interesting that the absence of IHC ERBB2 detection did not directly correlate with increased 
cold ischemia time, as three of the four IHC-negative but ISH-amplified cases had a cold ischemia time that was within current recommendations $(<1 \mathrm{~h})$. It is also interesting that in the $<1 \mathrm{~h}$ cold ischemia time, application of ASCO/CAP single-probe scoring guidelines resulted in 100\% concordance (no gain or loss of ERBB2 status) between FISH, DDISH, and IHC. In the three cases that showed discrepant results between scoring methods in the $<1 \mathrm{~h}$ cold ischemia time group, one was equivocal by FISH (ASCO/CAP single-probe guidelines) and IHC. The two remaining discrepant cases were called nonamplified by ASCO/CAP single-probe guidelines and amplified by both ASCO/CAP dual probe and FDA in-situ hybridization scoring guidelines. It is also remarkable that two of the discrepant cases demonstrated aneusomy or near aneusomy for chromosome 17 (CEP17 counts of 1.2 and 1.3 in cases 3 and 4, respectively) (Supplementary Table 1). In addition, these two cases showed low levels of $E R B B 2$ amplification (ERBB2 copy number of 3 by FISH and DDISH for case 3 with an ERBB2/ CEP17 ratio of 2.5 and 2.6 by FISH and DDISH, respectively; ERBB2 copy number of 3.1 by FISH and DDISH for case 4 with an ERBB2/CEP17 ratio of 2.3 and 2.4 by FISH and DDISH, respectively). Therefore, given the low level of ERBB2 copy number and the presence of low level CEP17 aneusomy, it is quite possible that these cases represent false positives based on ratio scoring. Given these findings, we conclude that the discordant results observed by ISH methods at the $<1 \mathrm{~h}$ cold ischemia time are attributable to scoring guideline selection and aneusomy rather than to an effect of cold ischemia time.

In the entire cold ischemia time cohort $(n=84)$, only two cases showed a major discordance in which ERBB2 was identified as overexpressed by ISH and non-amplified by IHC (Table 1; cold ischemia time $<1 \mathrm{~h}$; cases 3 and 4 ). Interestingly, these two major discordances occurred in the two cases mentioned above with CEP17 aneusomy present. Therefore, in relation to cases with a change in amplified/overexpressed status, overall concordance between IHC and ISH was seen in 82 of 84 cases $(98 \%)$, which is in line with published discordance rates. ${ }^{12}$ In addition to the reasons mentioned above, discrepancies between IHC and ISH results in our study could have occurred for multiple reasons. One possibility is loss of epitope specificity due to degradation secondary to a lack of prompt fixation (ie, an effect of cold ischemia time). A second factor could be the inherent limitations of IHC-based detection. ${ }^{24-26}$ On the basis of the fact that three of the four major discrepancies occurred within the $<1 \mathrm{~h}$ cold ischemia time cohort (the shortest cold ischemia time evaluated and a time that falls within the updated ASCO/CAP guidelines as acceptable for cold ischemia time), we think that this discrepancy in ERBB2 testing is more likely a limitation of IHC as compared with ISH and is not directly related to a cold ischemia time. ERBB2 scoring guidelines also play a role, as the correlation between amplified ISH and $3+$ IHC cases achieved higher concordance using the ASCO/CAP singleprobe guidelines.

For minor discordances (discrepancy involving alteration in non-amplified or equivocal ERBB2 status), the rate of IHC discordances were higher than that for FISH and DDISH. However, in the ISH and IHC discordant cases, the result by IHC was frequently equivocal, thus a reflex ISH assay would have been performed to resolve the ERBB2 status. Therefore, because of reflex testing, no practical and clinically significant effect was observed by increasing cold ischemia time.

Most prior studies that have tried to address the role of cold ischemia time in ERBB2 testing have utilized small patient populations and have artificially manipulated/exaggerated the time to formalin fixation. ${ }^{18,22}$ In this study, we accurately tracked cold ischemia time on actual patient specimens without artificial manipulation. In taking this approach, we demonstrate the actual sample handling at a single large institution, and show that only a subset of the samples $(N=46)$ meet the new ASCO/CAP guideline of $<1 \mathrm{~h}$ cold ischemia time with the remainder of the specimens having a cold ischemia time between $2-6 \mathrm{~h}$. This study design for assessing an effect of cold ischemia time is likely more reflective of real world sample handling then the artificial model systems that are currently published for cold ischemia time and prolonged formalin fixation for breast biomarkers ERBB2, ER, and PR. ${ }^{17,18,27}$ One disadvantage of this experimental design/approach is that cold ischemia time points beyond $3 \mathrm{~h}$ in clinical practice are rare and our $>3 \mathrm{~h}$ cold ischemia time cohort contained no ERBB2-amplified or overexpressed cases. Given the retention of strong internal control hybridization signals with CEP17 and ERBB2 signals by ISH in both tumor and surrounding stromal cell nuclei, we are certain that the six cases in the $>3 \mathrm{~h}$ cold ischemia time frame are truly ERBB2 negative. However, without a larger population or a selected population with known ERBB2-positive status, we are unable to provide conclusive evidence on cold ischemia time beyond $3 \mathrm{~h}$. Further studies including a larger number of patients or a prescreened ERBB2positive population will be needed to address the effects of cold ischemia time beyond $3 \mathrm{~h}$.

One additional limitation in our experimental model is the lack of sequential testing in the same patient. Rather than examining the same patient's tissue at multiple time points, we evaluated a unique patient sample at each cold ischemia time point. Therefore, by design, we could not follow-up with sequential testing on each patient at varying cold ischemia time points. Our method does not allow direct detection of the time point when ERBB2 signal detection is lost in an individual patient. However, we were able to include a large patient 
population and show actual specimen handling data at a large institution. In addition, our experimental model was made possible through the utilization of ISH techniques, which by design include a nonERBB2 hybridization control probe (chromosome 17; CEP17) that serves as a positive control for hybridization and could be detected in both tumor and stromal cell nuclei in all cases. In this study, all cases contained a non-ambiguous CEP17 and ERBB2 probe signal; therefore, the presence or absence of ERBB2 amplification could be accurately assessed for each patient.

Although there was nearly $100 \%$ concordance in $E R B B 2$ scores generated by FISH and DDISH, there was a significant difference in the signal intensity and signal preservation between these two methodologies. Using a four point scale for intensity of ERBB2 and CEP17 in both tumor and stroma, DDISH showed a higher average intensity score than FISH over all cold ischemia time points $(P=<0.05)$. In addition to having a higher overall intensity score (ie, easier signal to detect), the degradation of signal intensity following increased cold ischemia time was significant for FISH $(P=<0.05)$ but not DDISH $(P=$ not significant). This datum suggests that although no amplified case was missed by FISH or DDISH in cold ischemia times up to $3 \mathrm{~h}$, the ability to identify and score DDISH signal was superior to FISH and the trend of diminishing signal intensity with increased cold ischemia time was accelerated using FISH compared with DDISH. Therefore, it is possible that time points greater than $3 \mathrm{~h}$ would show a difference in detection of ERBB2 amplification between DDISH and FISH, however, a larger study cohort with increased cold ischemia time points would be needed to validate this observation.

In this study, which to our knowledge is the largest to date examining the effect of the preanalytical variable of cold ischemia time, we show that cold ischemia time up to $3 \mathrm{~h}$ does not alter the detection of ERBB2 by FISH or DDISH. In addition, the best correlation between IHC and ISH were observed using the ASCO/CAP single-probe guidelines. Using these guidelines, six cases were identified as amplified by FISH and DDISH, whereas five cases were identified as positive $(3+)$ by IHC. The one discrepant IHC case was scored as equivocal and therefore would have been reflexed to an ISH method. Therefore, detection of ERBB2 status by FISH, DDISH, and IHC achieved $100 \%$ concordance at all cold ischemia time points using ASCO/CAP single-probe scoring guidelines.

The findings of this study have practical implications that could affect specimen handling and fixation guidelines. The result of this and other recently published studies challenges the $\leq 1 \mathrm{~h}$ cold ischemia time guideline suggested by ASCO/CAP with respect to ERBB2 testing. In addition, it could be beneficial to perform a re-review of the three published in-situ hybridization guidelines for HER2 scoring.

\section{Acknowledgements}

This project has been funded in whole or in part with federal funds from the National Cancer Institute, National Institutes of Health, under Contract No. HHSN261200800001E.

\section{Disclosure/conflict of interest}

DLR is a stockholder in and consultant to HistoRx, the exclusive licensee to the Yale owned AQUA technology. DGH is a member of a speakers bureau for Genentech BioOncology. RRT receives honoraria for speaking on behalf of Ventana. The remaining authors have no conflict of interest.

\section{Disclaimer}

The content of this publication does not necessarily reflect the views or policies of the Department of Health and Human Services, nor does mention of trade names, commercial products, or organizations imply endorsement by the US Government.

\section{References}

1 Baselga J, Norton L, Albanell J, et al. Recombinant humanized anti-HER2 antibody (Herceptin) enhances the antitumor activity of paclitaxel and doxorubicin against HER2/neu overexpressing human breast cancer xenografts. Cancer Res 1998;58:2825-2831.

2 Paik S, Kim C, Wolmark N. HER2 status and benefit from adjuvant trastuzumab in breast cancer. N Engl J Med 2008;358:1409-1411.

3 Slamon DJ, Leyland-Jones B, Shak S, et al. Use of chemotherapy plus a monoclonal antibody against HER2 for metastatic breast cancer that overexpresses HER2. N Engl J Med 2001;344:783-792.

4 Pietras RJ, Pegram MD, Finn RS, et al. Remission of human breast cancer xenografts on therapy with humanized monoclonal antibody to HER-2 receptor and DNA-reactive drugs. Oncogene 1998;17:2235-2249.

5 Pegram M, Hsu S, Lewis G, et al. Inhibitory effects of combinations of HER-2/neu antibody and chemotherapeutic agents used for treatment of human breast cancers. Oncogene 1999;18:2241-2251.

6 Dang C, Fornier M, Sugarman S, et al. The safety of dose-dense doxorubicin and cyclophosphamide followed by paclitaxel with trastuzumab in HER-2/ neu overexpressed/amplified breast cancer. J Clin Oncol 2008;26:1216-1222.

7 Perez EA, Suman VJ, Davidson NE, et al. Cardiac safety analysis of doxorubicin and cyclophosphamide followed by paclitaxel with or without trastuzumab in the North Central Cancer Treatment Group N9831 adjuvant breast cancer trial. J Clin Oncol 2008;26: 1231-1238.

8 Tan-Chiu E, Yothers G, Romond E, et al. Assessment of cardiac dysfunction in a randomized trial comparing doxorubicin and cyclophosphamide followed by paclitaxel, with or without trastuzumab as adjuvant therapy in node-positive, human epidermal growth 
factor receptor 2-overexpressing breast cancer: NSABP B-31. J Clin Oncol 2005;23:7811-7819.

9 Liberato NL, Marchetti M, Barosi G. Cost effectiveness of adjuvant trastuzumab in human epidermal growth factor receptor 2-positive breast cancer. J Clin Oncol 2007;25:625-633.

10 Millar JA, Millward MJ. Cost effectiveness of trastuzumab in the adjuvant treatment of early breast cancer: a lifetime model. Pharmacoeconomics 2007;25:429-442.

11 Hillner BE, Smith TJ. Do the large benefits justify the large costs of adjuvant breast cancer trastuzumab? J Clin Oncol 2007;25:611-613.

12 Wolff AC, Hammond ME, Schwartz JN, et al. American Society of Clinical Oncology/College of American Pathologists guideline recommendations for human epidermal growth factor receptor 2 testing in breast cancer. Arch Pathol Lab Med 2007;131:18-43.

13 Updated April 2010; ASCO/CAP HER2 Testing Guidelines. Available at (http://www.cap.org/apps/docs/committees/ immunohistochemistry/clinical_notice.pdf).

14 Apple S, Pucci R, Lowe AC, et al. The effect of delay in fixation, different fixatives, and duration of fixation in estrogen and progesterone receptor results in breast carcinoma. Am J Clin Pathol 2011;135:592-598.

15 Arber DA. Effect of prolonged formalin fixation on the immunohistochemical reactivity of breast markers. Appl Immunohistochem Mol Morphol 2002;10: 183-186.

16 Goldsmith JD, Allred DC, Beasley MB, et al. Fixation time does not affect expression of HER2/neu. Am J Clin Pathol 2011;135:484 (author reply 485).

17 Ibarra JA, Rogers LW. Fixation time does not affect expression of HER2/neu: a pilot study. Am J Clin Pathol 2010;134:594-596.

18 Khoury T, Sait S, Hwang H, et al. Delay to formalin fixation effect on breast biomarkers. Mod Pathol 2009;22:1457-1467.
19 Selvarajan S, Bay BH, Choo A, et al. Effect of fixation period on HER2/neu gene amplification detected by fluorescence in situ hybridization in invasive breast carcinoma. J Histochem Cytochem 2002;50:1693-1696.

20 Willmore-Payne C, Metzger K, Layfield LJ. Effects of fixative and fixation protocols on assessment of Her-2/ neu oncogene amplification status by fluorescence in situ hybridization. Appl Immunohistochem Mol Morphol 2007;15:84-87.

21 Young SC, Burkett RJ, Stewart C. Discrepancy in ER levels of breast carcinoma in biopsy vs mastectomy specimens. J Surg Oncol 1985;29:54-56.

22 Moatamed NA, Nanjangud G, Pucci R, et al. Effect of ischemic time, fixation time, and fixative type on HER2/neu immunohistochemical and fluorescence in situ hybridization results in breast cancer. Am J Clin Pathol 2011;136:754-761.

23 Hicks DG, Kushner L, McCarthy K. Breast cancer predictive factor testing: the challenges and importance of standardizing tissue handling. J Natl Cancer Inst Monogr 2011; 2011;43-45.

24 Gown AM. Current issues in ER and HER2 testing by IHC in breast cancer. Mod Pathol 2008;21(Suppl 2): S8-S15.

25 Gown AM, Goldstein LC, Barry TS, et al. High concordance between immunohistochemistry and fluorescence in situ hybridization testing for HER2 status in breast cancer requires a normalized IHC scoring system. Mod Pathol 2008;21:1271-1277.

26 Khoury T, Kulkarni S, Morrison C, et al. HER2 testing: how to reach a high concordance rate between immunohistochemistry and fluorescence in situ hybridization. Appl Immunohistochem Mol Morphol 2011;19:424-430.

27 Ibarra JA, Rogers LW, Kyshtoobayeva A, et al. Fixation time does not affect the expression of estrogen receptor. Am J Clin Pathol 2010;133:747-755.

Supplementary Information accompanies the paper on Modern Pathology website (http://www.nature.com/ modpathol) 